\title{
Causes of extreme fatigue in underperforming athletes - a synthesis of recent hypotheses and reviews
}

\author{
P J Robson-Ansley (PhD) ${ }^{1}$ \\ L Lakier Smith (PhD) ${ }^{2}$ \\ ${ }^{1}$ Institute of Biomedical and Biological Sciences, Department of Sport and Exercise Sciences, University of Portsmouth, UK \\ ${ }^{2}$ Tshwane University of Technology, Pretoria
}

\begin{abstract}
The underperformance syndrome (UPS), previously known as the overtraining syndrome (OTS), has been defined as a persistent decrement in athletic performance capacity despite 2 weeks of relative rest. Clinical research has suggested that cytokines play a key role in fatigue in disease and chronic fatigue syndrome. Furthermore, it has recently been demonstrated that exogenous administration of interleukin-6 (IL-6) increases the sensation of fatigue during exercise. In light of current cytokine and chronic fatigue syndrome research, this article reviews and updates the cytokine theories that attempt to explain the aetiology of the debilitating fatigue experienced in OTS/UPS. Initially, it was proposed that UPS may be caused by excessive cytokine release during and following exercise, causing a chronic inflammatory state and 'cytokine sickness'. More recently, the hypothesis was extended and it was proposed that time-dependent sensitisation could provide a model through which the aetiology of UPS may be explained. According to this model, the principal abnormal factor in UPS is an intolerance/heightened sensitivity to IL-6 during exercise.
\end{abstract}

\section{Classical overtraining syndrome}

The Olympic motto citius, altius, fortius (faster, higher, stronger) epitomises the goal of athletic training. As athletes strive to improve their performance, inevitably their training load increases. Frequently their training strategies are successful and their performance improves. However, substantial anecdotal as well as experimental evidence ${ }^{17,18}$ links intensified

\section{CORRESPONDENCE:}

\section{P J Robson-Ansley}

Institute of Biomedical and Biological Sciences

Department of Sport and Exercise Sciences

University of Portsmouth

Portsmouth

PO1 2ER

United Kingdom

Tel: +442392848484

Fax: +442392842641

E-mail: paula.ansley@port.ac.uk training, for an extended period, with a chronic decrement in athletic performance. This reduced performance may manifest as an athlete failing to improve/maintain his/her previous performances, despite undergoing more intensive training, or an athlete reporting an inability to regain previous form following a vigorous competition.

Athletes must undergo significant amounts of physiological as well as psychological stress during training in order to provide sufficiently potent stimuli for appropriate adaptations to occur. To ensure that the athlete adapts favourably to the training load imposed, adequate rest is a crucial part of any training programme. If rest is not sufficient and the exercise stress alone or combined with other stressors (health, nutritional, environmental or psychological) is too great, the athlete may fail to adapt (maladapt) and enter a state of 'overreaching'. If the athlete continues training with insufficient rest, and in addition is exposed to further stressors (mentioned above), then a state of chronic underperformance may occur. This condition has been referred to as overtraining syndrome (OTS), but has also been referred to as staleness or burnout syndrome. ${ }^{22}$

It should be noted that although the condition of 'overreaching' may be a precursor to overtraining, certain coaches and athletes regard this condition as an integral part of training to achieve a supercompensatory effect. ${ }^{22}$ However, if an athlete is overreached, approximately 2 weeks of rest should result in a return to previous performance levels or even improved performance. ${ }^{22}$ In contrast, the OTS athlete will not recover in 2 weeks. It may take months for this athlete to recover, and in some instances the individual may never recover. ${ }^{22}$

OTS is commonly reported to occur in around $10-20 \%$ of elite endurance athletes ${ }^{32}$ and affects up to $65 \%$ of longdistance runners at some point in their athletic career. ${ }^{28}$ This condition should therefore not be regarded as a marginal problem but as an important and frequent event in the athletic community. ${ }^{22}$

\section{Redefinition of overtraining syndrome}

Although the aetiology of OTS remains elusive the term 'overtraining syndrome' may be a misnomer, since it implies that exercise is the sole causative factor of the syndrome. The recent redefinition of the syndrome focuses not only on the outcome, but also implies that the cause may be multi- 

factorial $^{39}$ being triggered by an accumulation of either sport and/or non-sport-related stress. ${ }^{29,43}$ Factors associated with the development of OTS may include extremely high loads of training with insufficient rest ${ }^{18}$ and/or excessive psychological stress ${ }^{29}$ and/or illness. ${ }^{29}$ In order to reflect the multidimensional nature of OTS, it is proposed that the broader term 'underperformance syndrome' (UPS) be adopted instead of OTS. ${ }^{7}$ Similar to the previous definition, UPS is defined as a persistent decrement in athletic performance, despite 2 weeks of relative rest, confirmed by both coach and athlete. ${ }^{7}$ The primary difference in the terminology is that UPS focuses on the final outcome of the condition, namely underperformance, whereas OTS focused on a single causative factor, namely the training load. UPS is the acronym that will be used in this article.

\section{Behavioural and mood-related signs and symp- toms of UPS}

Fatigue appears to be the most prominent and debilitating symptom of UPS. ${ }^{12}$ Specific fatigue-related symptoms reported by UPS athletes include feeling continuously tired despite adequate rest, as well as waking tired and unrefreshed despite an adequate duration of undisturbed sleep. ${ }^{12}$ In addition to these generalised reports of fatigue, the athlete complains extensively of local fatigue usually in the exercising muscles, and frequently describes the feeling of 'heavy legs' during exercise. ${ }^{12}$

Apart from the common symptom of persistent fatigue, a plethora of fatigue-related signs and symptoms have been associated with UPS, as is comprehensively detailed by Fry et al. ${ }^{14}$ Predominant symptoms include: (i) general malaise/flu-like symptoms; (ii) reduced interest in training and competing; (iii) reduced concentration; (iv) reduced socialising; $(v)$ increased daytime sleepiness and waking unrefreshed; (vi) reduced appetite; and (vii) depression.

Many of the above symptoms may in fact be related to an underlying depression since many of these features, including fatigue, reflect classic neurovegetative features of depression. ${ }^{1}$ However, the issue of whether or not the UPS athlete is suffering from depression per se, remains somewhat controversial. Morgan and colleagues ${ }^{31}$ have suggested that many UPS athletes suffer from what appears to be clinical depression and that frequently depression is the first sign of UPS, but is often ignored if the athlete is still performing adequately. In support of the presence of depression, Armstrong and VanHeest ${ }^{2}$ have suggested that this could well be the underlying cause of OTS and recommend the use of antidepressants to treat UPS. Uusitalo et al. ${ }^{46}$ also support the notion of depression being associated with UPS.

It has also been postulated ${ }^{6,12}$ that clinical depression is not a commonly reported sign of UPS. These researchers have postulated that when depression is present, rather than being a cause of UPS, it may occur at a later stage as a consequence of UPS, due to the frustration the athlete experiences as a consequence of underperformance.

Thus many questions exist concerning the association between UPS and depression. Questions include whether or not depression actually occurs, and if it does, what is the time point of onset of depression (does this precede the reduction in performance or is it a consequence); and what is the degree of depression (mild/moderate/severe). An additional issue is whether or not depression is a separate symptom or part of a more extensive syndrome, 'sickness behaviour', ${ }^{41}$ which will be discussed in the next section. ${ }^{19,37}$ Lastly, important from the perspective of this article is that if depression does exist, is the fatigue experienced during UPS part of the depressed symptomology or is the fatigue a symptom in and of itself.

\section{Communication of the sensation of fatigue from body to brain}

\section{The cytokine hypothesis of overtraining syndrome}

The universal and most debilitating symptom in UPS is the persistent fatigue reported by athletes. A hypothesis that attempts to explain some of these fatigue-associated mood/ behaviour changes is the "cytokine hypothesis of overtraining'. ${ }^{43}$ This proposes that intense and frequent training/competing, over an extended period, with insufficient rest/recovery time, may result in exercise-induced tissue trauma. It was further suggested that this diffuse tissue trauma may evoke a chronic, systemic inflammatory response. ${ }^{13}$ Chronic inflammation is a complex event and, amongst other sequelae, there is increased synthesis of pleiotropic proteins, viz. cytokines, produced by many eukaryotic cells. Cytokines may be grouped into several different families based on structure and/or function, such as anti- and pro-inflammatory cytokines. The pro-inflammatory cytokines, which appear to be associated with chronic inflammation, include interleukin (IL) $-1 \beta$, IL-6 and/or tumour necrosis factor- $\alpha$ (TNF $\alpha){ }^{13}$ It was further hypothesised that elevated circulating levels of any of these circulating cytokines could account for the many previously noted signs and symptoms of UPS, since cytokines have been shown to trigger alterations in immune, metabolic, physiological and behavioural responses. ${ }^{9,10,13}$ Although it was suggested that the primary source of serum cytokines was due to tissue trauma, it was also acknowledged that other factors such as illness, could initiate elevations in peripheral cytokine levels.

For a long while researchers have known that 'information' from the central nervous system (CNS) can be transmitted to the periphery of the body, via the sympathetic nervous system and/or the hypothalamic pituitary axis. ${ }^{27}$ However, the manner in which peripheral body-events, such as infection or injury, can 'inform' the CNS, has only recently been elucidated. It is now clear that cytokines function as messenger molecules that can access the CNS from the periphery. This may occur via neural and/or humoral routes. ${ }^{10}$ It should be noted that cytokines may also be produced in the CNS and subsequently released into the peripheral circulation. ${ }^{41}$ This circular, interconnected, reverberating mind-body interaction forms the basis of psychoneuroimmunology. ${ }^{41}$

Once within the CNS, IL-1 $\beta$, IL- 6 and TNF $\alpha$ are capable of activating specific brain structures, resulting in the 
manifestation of a constellation of behaviours referred to as 'sickness behaviour' or 'recuperative behaviour'. ${ }^{19,37}$ This cluster of behaviours includes the following signs and symptoms: weakness, malaise, listlessness, and inability to concentrate. ${ }^{19,37}$ Additionally, individuals are somewhat depressed and lethargic, showing little interest in their surroundings. ${ }^{19,37}$ Much of this is similar to what has been described in UPS. ${ }^{14}$

Similarly to what Hans Selye described as a generalised stress response, which occurs in response to a variety of different stressors, ${ }^{42}$ this sickness behaviour may be regarded as a generalised response to illness and/or injury, and may also be induced by a variety of pathogens and/or tissue trauma. Initially sickness behaviour was regarded as an unimportant, inconvenient by-product of sickness/injury. However, it is now recognised that it represents a highly organised, motivated strategy used to fight infection/illness so as to optimise healing/recovery from trauma. ${ }^{19,37}$

In summary, the cytokine hypothesis suggests that any of the pro-inflammatory cytokines (IL-1 $\beta$, and/or IL-6 and/ or $\mathrm{TNF} \alpha$ ) that may be produced by excessive training with insufficient rest and subsequent tissue trauma, may be responsible for many of the psychological changes including the extreme fatigue seen in UPS.

\section{Interleukin-6 fatigue hypothesis of UPS}

Although the cytokine hypothesis of OTS could account for different aetiologies of OTS, such as psychological stress or Epstein-Barr virus, ${ }^{43}$ the emphasis was on excessive training/competing with insufficient rest/recovery and subsequent tissue trauma being the primary initiator. Furthermore, the author proposed several pro-inflammatory cytokines being implicated in UPS, including IL- $1 \beta$ and TNF $\alpha$. Although these 2 cytokines may ultimately be implicated in UPS, substantially more research has been done pertaining to exercise and IL-6, as well as fatigue and IL-6, hence the 'interleukin- 6 hypothesis of UPS'. ${ }^{39}$ The IL- 6 hypothesis emphasises that factors aside from exercise-induced tissue trauma, trigger a dysregulated inflammatory response in UPS, causing either increased levels of circulating IL-6 or an increased sensitivity to IL-6. Additionally, this hypothesis stresses the central role of IL- 6 in the persistent fatigue experienced during UPS.

Apart from fatigue-inducing properties, IL- 6 has many functions and a wide range of biological activities, such as support of erythropoeisis, regulation of immune system responses, $^{20}$ and generation of acute-phase reactions. ${ }^{20}$ Recent research suggests that IL-6 may also play a metabolic role inducing counterregulatory hormones, hepatic glucose output and lipolysis (reviewed elsewhere ${ }^{35,36}$ ). Furthermore, IL-6 has been proposed as a glucose-regulator during prolonged exercise. $^{45}$

\section{IL-6 induces the sensation of fatigue at rest}

Studies investigating the effect of IL-6 on resting healthy individuals showed that low doses of recombinant human IL-6 (rhIL-6, a synthesised form of IL-6) induce an increased sensation of fatigue. ${ }^{3,44}$ In one study subjects were given either a single low dose of IL-6 that mimicked systemic increases in this cytokine typically observed during a moderate infection (and also comparable with those measured in athletes following prolonged exercise ${ }^{33}$ ), or a placebo. ${ }^{44}$ A self-reported mood state questionnaire was completed 3 hours after IL6 administration; scores showed that subjects were feeling significantly more tired compared with the placebo trial. All signs and symptoms associated with the IL- 6 administration had resumed pre-trial values when assessed 12 hours later. Although clinicians readily acknowledge the fatigue-inducing properties of IL- $6,{ }^{21,23,48}$ these properties have gone largely unrecognised by exercise physiologists.

\section{Exogenous IL-6 administration exacerbates fatigue in healthy trained runners during exercise}

Recent data obtained from a performance-related study ${ }^{40}$ indicate that IL- 6 may also play a role in the sensation of fatigue during exercise. When a dose of rhlL- 6 was administered to subjects prior to a $10 \mathrm{~km}$ time trial (to induce equivalent plasma concentrations found following prolonged exercise or a moderate infection), subjects reported an increased sensation of physical and psychological fatigue during the exercise trial that ultimately resulted in a significant decrement in performance. This led the researchers to suggest that IL-6 may also act as a circulating fatigue-inducing molecule during exercise. Both global mood state and fatigue worsened as scores were significantly higher immediately post-exercise in the IL- 6 trial compared with placebo. The effect of the IL- 6 treatment was relatively short-lived, as by approximately 3 hours post-IL-6 administration, scores for global mood state and fatigue ${ }^{16}$ had returned to pre-treatment values. It appears that transiently elevated levels of IL- 6 negatively affect the sensation of fatigue and global mood state in healthy runners.

Rating of perceived exertion ${ }^{5}$ was also measured throughout the exercise trials but was not different between trials despite the subjects running slower during the IL-6 trial (Robson-Ansley et al. - unpublished data, 2003). This may be perceived as subjects running with the 'same pain but less gain' compared with the placebo trial. Subjects felt they were running with the same effort but were, in fact, running significantly slower; some subjects reported that they could not get their legs moving and complained of 'heavy legs' during the exercise trial. This is the first study to directly identify that an elevated plasma IL-6 concentration (similar to that measured following a prolonged bout of exercise) has fatigue-inducing properties that can affect both mood state and athletic performance, both of which are factors associated with UPS.

\section{Heightened sensitivity to IL-6 in chronic fatigue syn- drome - the missing link in UPS?}

UPS has several parallels with chronic fatigue syndrome (CFS), in particular persistent fatigue and mood disturbances, ${ }^{12}$ hence research findings on CFS may aid elucidation of the aetiology of UPS. Resting plasma IL-6 levels appear similar between healthy athletes and UPS athletes, ${ }^{34}$ as well as between healthy individuals and CFS patients. Whether 
or not the IL-6 response to exercise differs between healthy and UPS athletes is currently undetermined. Although resting levels of IL-6 in healthy and chronically fatigued individuals may be comparable, recent research highlights the fact that CFS patients have a heightened sensitivity to IL-6 compared with normal, healthy individuals. ${ }^{3,15}$ Following IL-6 administration, CFS patients experienced a greater number of somatic symptoms such as aches, fatigue and malaise, with a shorter latency, compared with the healthy controls. ${ }^{3}$ The feelings of fatigue and malaise remained up to 24 hours after the rhIL-6 administration in the CFS group. Unfortunately, initial levels of IL-6 were not reported for the patients with CFS or normal control subjects, so it is not known whether CFS patients commenced the study with elevated plasma IL6 levels. The cause of the abnormal/heightened response to rhIL-6 in CFS is unclear, but elucidation of this may provide insight into the chronic fatigue during exertion experienced by athletes with UPS.

\section{Heightened sensitivity to IL- 6 in UPS}

The IL-6 hypothesis ${ }^{39}$ proposes that time-dependent sensitisation (TDS) to IL-6, similar to what has been proposed for CFS patients, could provide a model through which the aetiology of UPS may be explained. TDS is described as a progressive and persistent amplification of behavioural and biochemical responses to repeated intermittent stimuli over time. ${ }^{4}$ The model proposes that a long-term or permanent physiological adaptation occurs as a consequence of initial exposure to a severe physical or psychological stimulus (or stimuli). This results in the individual developing a progressive and amplified intolerance on repeated exposure rather than developing a tolerance. In accordance with the TDS model, the IL- 6 hypothesis proposes that initial stressors or trigger factors for UPS such as severe infection, ${ }^{47}$ excessive exercise, heat stroke ${ }^{8}$ or severe psychological stress ${ }^{25,26}$ evoke a substantial cytokine response. These proposed trigger factors may sensitise individuals in such a manner that they become sensitive to IL-6 and exercise-intolerant (maladapting to the exercise stress), causing performance decrements with each subsequent training session (Fig. 1).

The exact physiological mechanism affected by the initial exposure to a severe stressor is unknown, but it is thought that stressors and/or the biochemical responses to the stressors could induce gene transcription factors. ${ }^{38}$ Activation of these gene transcription factors by exposure to a significant stressor could cause a long-term alteration of responsivity to future stressors, possibly by increasing cytokine receptor densities or soluble receptor concentrations. In support of this, one study $^{30}$ demonstrated that repeated exposure to stress induces changes in IL-6 mRNA and IL-6 receptor mRNA in the brain, which are different from those induced during a single exposure to stress.

Alternatively, the initial trigger factor(s) that stimulated the TDS may have caused permanent or long-term damage to the blood-brain barrier (BBB). The integrity of the BBB permeability to cytokines can be altered by exposure to factors such as excessive cytokine exposure, ${ }^{11}$ heat stress ${ }^{49}$ and endotoxins. ${ }^{24}$ It is conceivable that this increased

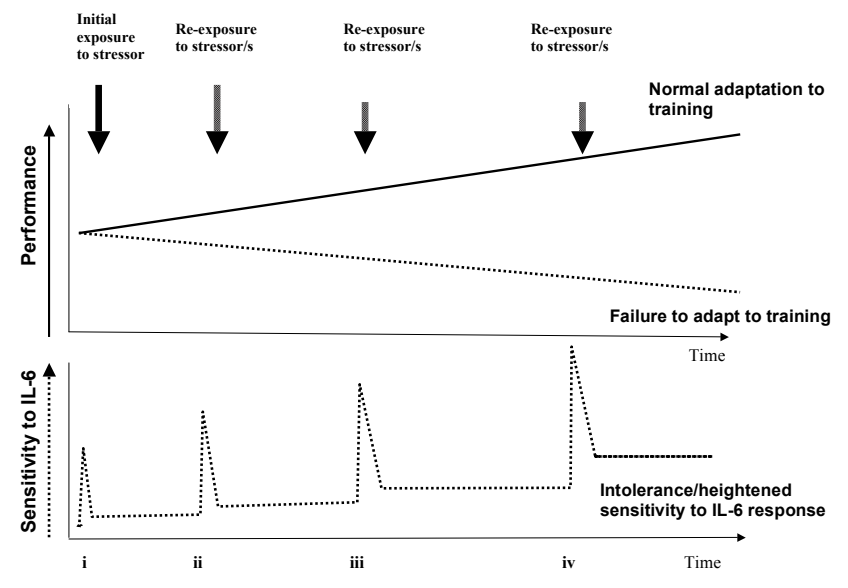

Fig. 1. Interleukin-6 fatigue hypothesis of underperformance syndrome. A schematic diagram of the time-dependent sensitisation phenomenon (adapted with permission from Robson ${ }^{39}$. $i=$ initial exposure to stressor induces long-term change in blood-brain barrier integrity and/or c-fos expression; ii, iii, iv = further exposure to stressor/s triggers an intolerance/heightened sensitivity to IL-6 causing further performance deterioration).

permeability to cytokines may induce a heightened degree of 'sickness behaviour' and associated fatigue, upon a subsequent exposure that could be evoked by factors such as prolonged exercise.

The IL- 6 hypothesis of UPS suggests that the relentless fatigue experienced by these athletes is due to stimulation of certain CNS structures by an increase in or heightened sensitivity to IL-6. Present research suggests that resting blood levels of IL- 6 in UPS athletes are similar to levels in healthy controls; there is minimal evidence on post-exercise levels of IL- 6 in UPS athletes. The possibility of increased sensitivity to IL-6 was proposed as a possible explanation for the extreme fatigue experienced by these athletes.

\section{Summary and conclusion}

Two hypotheses were presented in this article in an attempt to explain the debilitating fatigue experienced during UPS. The cytokine hypothesis ${ }^{43}$ stresses excessive training with resultant tissue damage and the potential role of several cytokines that could induce fatigue, and that are possibly associated with depression. The IL- 6 hypothesis ${ }^{39}$ focuses on a possible central role for this one cytokine, stressing multi-factorial causes of elevated IL-6 in UPS. This hypothesis further suggests that an increased sensitivity to IL-6 could be implicated in the extreme fatigue associated with UPS.

However, to date there is limited information to support these hypotheses. Thus it is acknowledged that extensive animal and human studies are warranted to either support or refute these suppositions.

\section{REFERENCES}

1. Anisman $\mathrm{H}$, Merali Z. Cytokines, stress, and depressive illness. Brain Behav Immun 2002; 16: 513-24. 

2. Armstrong LE, VanHeest JL. The unknown mechanism of the overtraining syndrome: clues from depression and psychoneuroimmunology. Sports Med 2002; 32: 185-209.

3. Arnold MC, Papanicolaou DA, O'Grady JA, et al. Using an interleukin-6 challenge to evaluate neuropsychological performance in chronic fatigue syndrome. Psychol Med 2002; 32: 1075-89.

4. Bell IR. White paper: Neuropsychiatric aspects of sensitivity to low-level chemicals: a neural sensitization model. Toxicol Ind Health 1994; 10: 277 312.

5. Borg GA. Psychophysical bases of perceived exertion. Med Sci Sports Exerc 1982; 14: 377-81.

6. Budgett R. Fatigue and underperformance in athletes: the overtraining syndrome. Br J Sports Med 1998; 32: 107-10.

7. Budgett R, Newsholme E, Lehmann M, et al. Redefining the overtraining syndrome as the unexplained underperformance syndrome. $\mathrm{Br} J$ Sports Med 2000; 34: 67-8.

8. Chung NK, Shabbir M, Lim CL. Cytokine levels in patients with previous heatstroke under heat stress. Mil Med 1999; 164: 306-10.

9. Dantzer R. Cytokine-induced sickness behaviour: a neuroimmune response to activation of innate immunity. Eur J Pharmacol 2004; 500: 399-411.

10. Dantzer R. How do cytokines say hello to the brain? Neural versus humoral mediation. Eur Cytokine Netw 1994; 5: 271-3.

11. de Vries HE, Blom-Roosemalen MC, van Oosten $\mathrm{M}$, et al. The influence of cytokines on the integrity of the blood-brain barrier in vitro. J Neuroimmunol 1996; 64: 37-43.

12. Derman W, Schwellnus MP, Lambert MI, et al. The 'worn-out athlete': a clinical approach to chronic fatigue in athletes. J Sports Sci 1997; 15: 341-51.

13. Feghali CA, Wright TM. Cytokines in acute and chronic inflammation. Front Biosci 1997; 2: d12-26.

14. Fry RW, Morton AR, Keast D. Overtraining in athletes. An update. Sports Med 1991; 12: 32-65

15. Gaab J, Rohleder N, Heitz V, et al. Stress-induced changes in LPS-induced pro-inflammatory cytokine production in chronic fatigue syndrome. Psychoneuroendocrinology 2005; 30: 188-98.

16. Grove JR, Prappavessis H. Preliminary evidence for the reliability and validity of an abbreviated Profile of Mood State questionnaire. Int J Sport Psychology 1992; 23: 93-109.

17. Halson SL, Lancaster GI, Achten J, Gleeson M, Jeukendrup AE. Effects of carbohydrate supplementation on performance and carbohydrate oxidation after intensified cycling training. J Appl Physiol 2004; 97: 1245-53.

18. Jeukendrup AE, Hesselink MK, Snyder AC, Kuipers H, Keizer HA. Physiological changes in male competitive cyclists after two weeks of intensified training. Int J Sports Med 1992; 13: 534-41.

19. Kelley KW, Bluthe RM, Dantzer R, et al. Cytokine-induced sickness behavior. Brain Behav Immun 2003; 17: Suppl 1, S112-8.

20. Kishimoto T. The biology of interleukin-6. Blood 1989; 74: 1-10.

21. Kurzrock R. The role of cytokines in cancer-related fatigue. Cancer 2001; 92: 1684-8.

22. Lehmann M, Foster C, Dickhuth HH, Gastmann U. Autonomic imbalance hypothesis and overtraining syndrome. Med Sci Sports Exerc 1998; 30: 1140-5.

23. Lloyd A, Gandevia S, Brockman A, Hales J, Wakefield D. Cytokine production and fatigue in patients with chronic fatigue syndrome and healthy control subjects in response to exercise. Clin Infect Dis 1994; 18: Suppl 1, S142-6.

24. Lustig S, Danenberg HD, Kafri Y, Kobiler D, Ben Nathan D. Viral neuroinvasion and encephalitis induced by lipopolysaccharide and its mediators. $J$ Exp Med 1992; 176: 707-12.

25. Maes M, Lin AH, Delmeire L, et al. Elevated serum interleukin-6 (IL-6) and IL-6 receptor concentrations in posttraumatic stress disorder following accidental man-made traumatic events. Biol Psychiatry 1999; 45: 833-9.

26. Maes M, Ombelet W, De Jongh R, Kenis G, Bosmans E. The inflammatory response following delivery is amplified in women who previously suffered from major depression, suggesting that major depression is accompanied by a sensitization of the inflammatory response system. J Affect Disord
2001; 63: 85-92.

27. Maier SF, Watkins LR. Cytokines for psychologists: implications of bidirectional immune-to-brain communication for understanding behavior, mood, and cognition. Psychol Rev 1998; 105: 83-107.

28. McKenzie DC. Markers of excessive exercise. Can J Appl Physiol 1999; 24: 66-73.

29. Meehan HL, Bull SJ, Wood DM, James DV. The Overtraining Syndrome: a multicontextual assessment. The Sport Psychol 2004; 18: 154-71.

30. Miyahara S, Komori T, Fujiwara R, et al. Effects of repeated stress on expression of interleukin-6 (IL-6) and IL- 6 receptor mRNAs in rat hypothalamus and midbrain. Life Sci 2000; 66: L93-8.

31. Morgan WP, Brown DR, Raglin JS, O'Connor PJ, Ellickson KA. Psychological monitoring of overtraining and staleness. Br J Sports Med 1987; 21: 107-14.

32. Morgan WP, Costill DL, Flynn MG, Raglin JS, O'Connor PJ. Mood disturbance following increased training in swimmers. Med Sci Sports Exerc 1988; 20: 408-14.

33. Ostrowski K, Rohde T, Zacho M, Asp S, Pedersen BK. Evidence that interleukin- 6 is produced in human skeletal muscle during prolonged running. $J$ Physiol 1998; 508: Pt 3, 949-53.

34. Parry-Billings $M$, Budgett $R$, Koutedakis $\mathrm{Y}$, et al. Plasma amino acid concentrations in the overtraining syndrome: possible effects on the immune system. Med Sci Sports Exerc 1992; 24: 1353-8.

35. Pedersen BK, Steensberg A, Fischer C, Keller C, Ostrowski K, Schjerling P. Exercise and cytokines with particular focus on muscle-derived IL-6. Exerc Immunol Rev 2001; 7: 18-31.

36. Pedersen BK, Steensberg A, Schjerling P. Exercise and interleukin-6. Curr Opin Hematol 2001; 8: 137-41.

37. Pollmacher T, Haack M, Schuld A, Reichenberg A, Yirmiya R. Low levels of circulating inflammatory cytokines - do they affect human brain functions? Brain Behav Immun 2002; 16: 525-32.

38. Post RM. Transduction of psychosocial stress into the neurobiology of recurrent affective disorder. Am J Psychiatry 1992; 149: 999-1010.

39. Robson P. Elucidating the unexplained underperformance syndrome in endurance athletes: the interleukin-6 hypothesis. Sports Med 2003; 33: 771-81.

40. Robson-Ansley PJ, de Milander L, Collins M, Noakes TD. Acute interleukin6 administration impairs athletic performance in healthy, trained male runners. Can J Appl Physiol 2004; 29: 411-8.

41. Schiepers OJ, Wichers MC, Maes M. Cytokines and major depression. Prog Neuropsychopharmacol Biol Psychiatry 2005; 29: 201-17.

42. Selye H, Fortier C. Adaptive reaction to stress. Psychosom Med 1950; 12 : 149-57.

43. Smith LL. Cytokine hypothesis of overtraining: a physiological adaptation to excessive stress? Med Sci Sports Exerc 2000; 32: 317-31.

44. Spath-Schwalbe E, Hansen K, Schmidt F, et al. Acute effects of recombinant human interleukin- 6 on endocrine and central nervous sleep functions in healthy men. J Clin Endocrinol Metab 1998; 83: 1573-9.

45. Steensberg A, van Hall G, Osada T, Sacchetti M, Saltin B, Klarlund PB. Production of interleukin- 6 in contracting human skeletal muscles can account for the exercise-induced increase in plasma interleukin-6. J Physiol 2000; 529: Pt 1, 237-42.

46. Uusitalo AL, Valkonen-Korhonen M, Helenius $\mathrm{P}$, Vanninen $\mathrm{E}$, Bergstrom $\mathrm{KA}$, Kuikka JT. Abnormal serotonin reuptake in an overtrained, insomnic and depressed team athlete. Int J Sports Med 2004; 25: 150-3.

47. Vallieres L, Rivest S. Interleukin- 6 is a needed proinflammatory cytokine in the prolonged neural activity and transcriptional activation of corticotropinreleasing factor during endotoxemia. Endocrinology 1999; 140: 3890-903.

48. Vgontzas AN, Papanicolaou DA, Bixler EO, Kales A, Tyson K, Chrousos GP. Elevation of plasma cytokines in disorders of excessive daytime sleepiness: role of sleep disturbance and obesity. J Clin Endocrinol Metab 1997; 82: 1313-6.

49. Wijsman JA, Shivers RR. Heat stress affects blood-brain barrier permeability to horseradish peroxidase in mice. Acta Neuropathol (Berl) 1993; 86: 49-54. 